\title{
ELASTIC-PLASTIC TORSION OF CIRCULAR RING SECTORS*
}

\author{
BY \\ W. FREIBERGER \\ Brown University
}

\begin{abstract}
The solution is presented to the problem of uniform torsion of circular ring sectors with circular cross section under the assumption of perfect plasticity. The elastic-plastic problem is solved by a semi-inverse method. When the entire ring is plastic a discontinuity of stress appears which may be regarded as the limiting case of an elastic core; such discontinuities have recently been discussed in the literature [1]. The solution for the discontinuous fully plastic stress distribution is exact, that for the elastic-plastic case approximate in the sense that it is found exactly for cross sections differing slightly from the circular. This difference is negligible for ratios of ring radius $R$ to cross section radius $\rho$ occurring in practical applications to helical springs of small pitch.
\end{abstract}

1. The completely plastic problem. The stress system being independent of the meridional coordinate $\theta$ the equations of equilibrium in cylindrical polar coordinates reduce to the single equation

$$
\frac{\partial\left(r^{2} \tau_{r}\right)}{\partial r}+\frac{\partial\left(r^{2} \tau_{s}\right)}{\partial z}=0,
$$

where $\tau_{r}$ and $\tau_{s}$ are the only non-vanishing stresses, viz. the shearing stresses transmitted across meridional planes. The yield conditions of Tresca or von Mises require that

$$
\tau_{r}^{2}+\tau_{s}^{2}=k^{2},
$$

where $k$ is the yield stress in simple shear assumed to be a constant throughout the body. The problem is completed by the boundary condition that the resultant shearing stress with the components $\tau_{r}$ and $\tau_{z}$ be tangential to the contour of the cross section.

To solve Eqs. (1) and (2) set

$$
\tau_{r}=k \cos \psi, \quad \tau_{z}=k \sin \psi,
$$

where $\psi$ is the angle between the stress vector and the positive $r$-direction; hence

$$
\frac{\partial \psi}{\partial r}-\cot \psi \frac{\partial \psi}{\partial z}-\frac{2}{r} \cot \psi=0 .
$$

This equation can be solved by the method of characteristics. The characteristic equations are

$$
\frac{d r}{d s}=\sin \psi, \quad \frac{d z}{d s}=-\cos \psi, \quad \frac{d \psi}{d s}=\frac{2}{r} \cos \psi .
$$

It follows from (3) and (5) that at any point the resultant shearing stress vector is normal to the characteristic through that point. In particular, the characteristics are normal to the stress-free boundary.

Integration of the first and second of Eqs. (5) gives

$$
r^{2} \cos \psi=c \text {, }
$$

*Received Sept. 21, 1955. 
where $c$ is a constant along a given characteristic. From (6) and the first two Eqs. (5) it follows that

$$
\frac{d z}{d r}=-\cot \psi=\mp\left\{\left(r / c^{1 / 2}\right)^{4}-1\right\}^{-1 / 2}
$$

or

$$
z=\mp(c / 2)^{1 / 2} F\left(45^{\circ}, \operatorname{arcos} c^{1 / 2} / r\right)+\text { constant, }
$$

where $F(\alpha, \varphi)$ is the incomplete elliptic integral of the first kind, in the notation of Ref. [2]. The constant in Eq. (7) is determined by the boundary condition along the stress-free surface; if $\beta$ is the angle between the positive $r$-direction and the radius vector from the center of the cross section to a boundary point $P$, we have $\tau_{r} \cos \beta+$ $\tau_{z} \sin \beta=0$, or, by Eq. (3), $\cos (\psi-\beta)=0$ and hence

$$
\sin \psi=\cos \beta \text { at } P \text {. }
$$

Moreover, at $P$,

$$
z=\rho \sin \beta, \quad r=R+\rho \cos \beta
$$

Hence, from Eqs. (6), (8), (9) the constant $c$ in Eq. (7) has the value

$$
c=(R+\rho \cos \beta)^{2} \sin \beta
$$

for the characteristic through boundary point $P(\rho \sin \beta, R+\rho \cos \beta)$. With this value the equation of the characteristic finally becomes

$$
\begin{aligned}
z / \rho=\sin \beta \mp 2^{-1 / 2}\left(\frac{R}{\rho}+\cos \beta\right) \sin ^{1 / 2} \beta\left\{F \left(45^{\circ}, \operatorname{arcos}\right.\right. & \frac{\left.(R+\rho \cos \beta) \sin ^{1 / 2} \beta\right)}{r} \\
& \left.-F\left(45^{\circ}, \operatorname{arcos} \sin ^{1 / 2} \beta\right)\right\} .
\end{aligned}
$$

The value of $\psi$, and hence that of the shearing stress components, on any point along the characteristic (11) is found from the equation

$$
\cos \psi=\left(\frac{R+\rho \cos \beta}{r}\right)^{2} \sin \beta .
$$

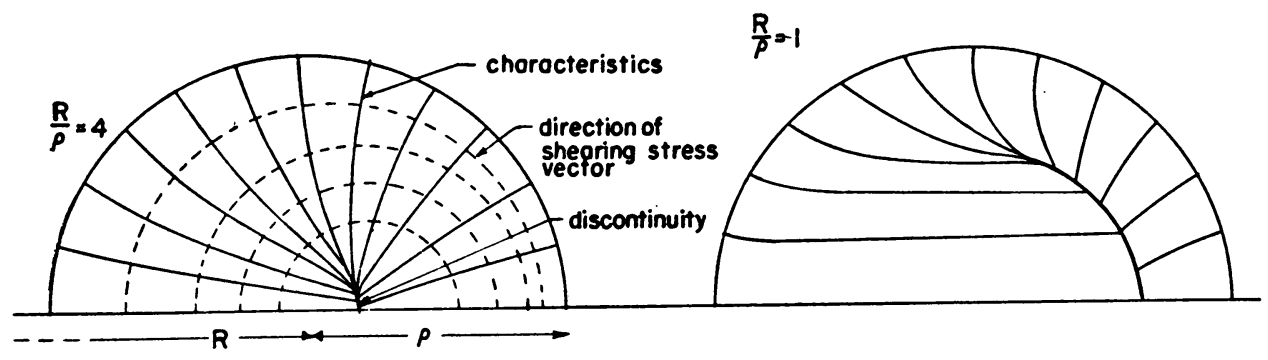

FIG. 1. Fully plastic stress distribution: characteristics and line of discontinuity for two ring sizes.

The characteristics intersect on a line of stress discontinuity. Equilibrium allows only the tangential component of the shearing stress to be discontinuous. The line of stress discontinuity must therefore bisect the angle formed by the characteristics meeting on this line. Figure 1 shows this situation for the rings specified by $R / \rho=1$ and $\mathrm{R} / \rho=4$. 
It can be shown (see [3], [4] and [5]) that the resultant of the considered stress system is indeed a force acting along the axis of the ring; the expression for this resultant is

$$
\frac{Z}{k \rho^{2}}=\iint\left\{1-\left(\frac{R}{\rho}+\cos \beta\right)^{4} \frac{\sin ^{2} \beta}{(r / \rho)^{4}}\right\}^{1 / 2} d\left(\frac{r}{\rho}\right) d\left(\frac{z}{\rho}\right),
$$

with the integration carried out over the cross section.

2. Velocities. The velocity-strains for the present problem obey the relations

$$
\begin{aligned}
& \frac{\partial v_{r}}{\partial r}=0, \quad \frac{\partial v_{z}}{\partial z}=0, \quad \frac{\partial v_{\theta}}{\partial \theta}+v_{r}=0, \quad \frac{\partial v_{z}}{\partial r}+\frac{\partial v_{r}}{\partial z}=0 \\
& \frac{1}{2}\left(\frac{1}{r} \frac{\partial v_{r}}{\partial \theta}+\frac{\partial v_{\theta}}{\partial r}-\frac{v_{\theta}}{r}\right)=\lambda \tau_{r}, \quad \frac{1}{2}\left(\frac{\partial v_{\theta}}{\partial z}+\frac{1}{r} \frac{\partial v_{z}}{\partial \theta}\right)=\lambda \tau_{z},
\end{aligned}
$$

which are satisfied by velocities of the form

$$
v_{r}=0, \quad v_{z}=K \theta, \quad v_{\theta}=\varphi(r, z),
$$

where $K$ is a constant and $\varphi(r, z)$ denotes the warping function. Thus,

$$
\frac{\partial \varphi}{\partial r}-\frac{\varphi}{r}=2 \lambda \tau_{r}, \quad \frac{\partial \varphi}{\partial z}+\frac{K}{r}=2 \lambda \tau_{s} .
$$

From (3) and (14) the differential equation of the warping function is seen to be

$$
\frac{\partial(\varphi / K)}{\partial r}-\cot \psi \frac{\partial(\varphi / K)}{\partial z}-\frac{\cot \psi+\varphi / K}{r}=0 .
$$

Equation (15) has the same characteristics in the $r, z$ plane as Eq. (4), and the variation of $\varphi$ along the characteristics is given by

$$
\frac{d(\varphi / K)}{d r}-\frac{\varphi / K}{r}=-\frac{1}{r} \frac{d z}{d r}=\mp_{r}^{c}\left(r^{4}-c^{2}\right)^{-1 / 2} .
$$

By integration one obtains:

$$
\begin{aligned}
\frac{\varphi}{K}=\mp \frac{r / \rho}{R / \rho+\cos \beta} \sin ^{-1 / 2} \beta\left\{2^{1 / 2} E(\right. & \left.45^{\circ}, \operatorname{arcos} \frac{R / \rho+\cos \beta}{r / \rho} \sin ^{1 / 2} \beta\right) \\
& \left.-2^{-1 / 2} F\left(45^{\circ}, \operatorname{arcos} \frac{R / \rho+\cos \beta}{r / \rho} \sin ^{1 / 2} \beta\right)\right\}+r C,
\end{aligned}
$$

where $F$ and $E$ are the incomplete elliptic integrals of first and second kind, respectively, and the constant $C$ is determined from the boundary condition on the line of discontinuity. Since the line of discontinuity is the limit of a narrow elastic core, the shear rate of the assumed rigid, plastic material must vanish along the line of discontinuity. Thus,

$$
\frac{\partial \varphi}{\partial r}=\frac{\varphi}{r}, \quad \frac{\partial \varphi}{\partial z}=-\frac{K}{r}
$$

and hence

$$
d \varphi=\frac{\varphi}{r} d r-\frac{K}{r} d z
$$


Since the circumferential velocity component $v_{0}$ is only determined to within an arbitrary additive constant, which corresponds to a rigid body rotation of the ring about the $z$ axis, the function $\psi$ may be given the value zero at some point on the discontinuity. Numerical integration of (15) then furnishes $\varphi$ along the discontinuity and so the required initial condition for integration along the characteristics originating on the discontinuity. Examples for this procedure are provided in Refs. [4] and [5].

3. The elastic-plastic problem. The solution to the elastic-plastic problem of a ring with approximately circular cross section will be obtained by an inverse method first proposed by Sokolowsky for the torsion of an elliptic cylinder [6].

It can be shown (see [7]) that the elastic torsion problem of circular rings requires the solution of the equation

$$
\left(\nabla^{2}-\frac{4}{r^{2}}\right) \frac{\Phi}{r^{2}}=\frac{1}{r^{2}}
$$

Here, $\Phi$ is a stress function in terms of which the stress components are expressed by

$$
\tau_{r}=\frac{A}{r^{2}} \frac{\partial \Phi}{\partial z}, \quad \tau_{z}=-\frac{A}{r^{2}} \frac{\partial \Phi}{\partial r},
$$

where $A$ is a constant affecting the absolute values but not the distribution of the stresses. For a given ring, this constant is proportional to the applied force.

Equation (17) can be written

$$
\frac{\partial^{2} \Phi}{\partial r^{2}}-\frac{3}{r} \frac{\partial \Phi}{\partial r}+\frac{\partial^{2} \Phi}{\partial z^{2}}=1
$$

If a solution of Eq. (19) can be found giving a stress distribution such that the yield condition Eq. (2) is satisfied along a more or less circular curve, then this curve may be taken as the elastic-plastic interface and used as the starting curve for the construction of the characteristics emanating from it. This is done by evaluating the ratio $\tau_{z} / \tau_{r}$ along the curve from the elastic solution and noting that the characteristics will be perpendicular to the direction of the vector $\left(\tau_{z}, \tau_{r}\right)$. Any orthogonal trajectory of the characteristics can be taken as the boundary of a cross section, for which an elastic, plastic stress distribution has thus been obtained. If two or more solutions of Eq. (17) give practically the same cross section, the progress of the plastic region with increasing external force can be traced. The stress function

$$
\Phi=\left(r^{2}-a^{2}\right)^{2}+b^{2} z^{2}
$$

is found to satisfy Eq. (19) if the constants $a$ and $b$ are related by the equation

$$
b^{2}=\frac{1-8 a^{2}}{2}
$$

Substituting Eq. (20) in Eq. (2) it follows that possible elastic-plastic boundaries are given by

$$
c^{2} z^{2}=r^{2}\left[\alpha^{4}-\left(r^{2}-\beta^{2}\right)^{2}\right]
$$

where

$$
c^{2}=b^{4} / 4, \quad \alpha^{4}=k^{2}\left(\frac{k^{2}}{16 A^{2}}+4 a^{2}\right) / 64 A^{2}, \quad \beta^{2}=\left(\frac{k^{2}}{4 A^{2}}+8 a^{2}\right) / 8,
$$


and $a$ and $b$ are related by Eq. (21). Clearly, $\beta^{4}-\alpha^{4}=a^{4}$. Figure 2 shows two elasticplastic interfaces; they are found from Eqs. (22) and (23) by giving the parameters the values $\alpha=1.5, \beta=3$ (interface 1) and $\alpha=1.1, \beta=3$ (interface 2), respectively. By drawing the characteristics based on these curves it is seen that the cross sectional boundary $R / \rho=6$ in Fig. 2 is approximately an orthogonal trajectory to the families based on either.

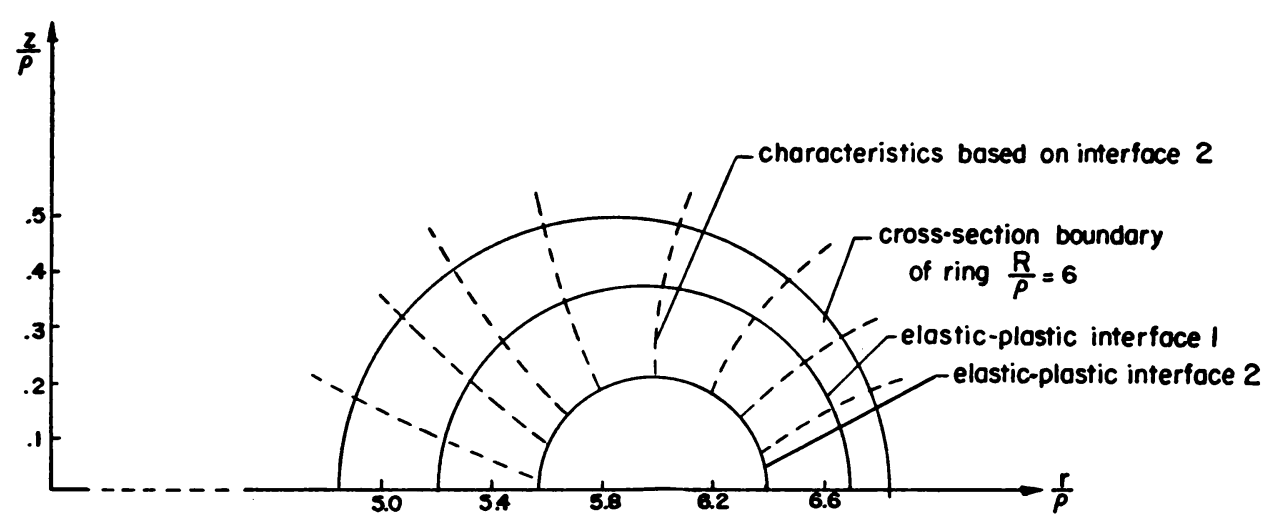

FIG. 2. Elastic-plastic solution. Showing boundary of ring considered and two elastic-plastic interfaces.

It remains to evaluate the resultant force corresponding to the fully elastic ring, the fully plastic ring, and the ring for which the elastic-plastic boundary has advanced to either of the two positions of Fig. 2. If the resultant force is denoted by Z,

$$
\begin{aligned}
\frac{Z}{k \rho^{2}} & =\frac{1}{k} \iint \tau_{z} d(r / \rho) d(z / \rho) \\
& =\iint \sin \psi d(r / \rho) d(z / \rho)-\frac{A}{k} \iint \frac{1}{r^{2}} \frac{\partial \Phi}{\partial r} d(r / \rho) d(z / \rho) \\
& =\iint \sin \psi d(r / \rho) d(z / \rho)-4 \frac{A}{k} \iint\left(r-\frac{a^{2}}{r}\right) d(r / \rho) d(z / \rho),
\end{aligned}
$$

where the first integral is taken over the plastic, the second over the elastic part of the cross section. Both integrals are best evaluated numerically by dividing the cross section into a net of squares and summing the values of the integrands at the centers of these squares.

$$
\begin{array}{rll}
\text { For } \alpha=1.5, \beta=3 & \text { one finds } & a^{2}=8.71, A / k=.329 \\
\text { for } \alpha=1.1, \beta=3
\end{array} \quad \begin{aligned}
& a^{2}=8.96, A / k=.625
\end{aligned}
$$

The results of the integrations are the following:

Ring fully elastic, with yield value just reached

at point of maximum stress:

$Z / k \rho^{2}=.2$

Ring with elastic-plastic boundary 1:

$Z / k \rho^{2}=.22$

Ring with elastic-plastic boundary 2 :

$Z / k \rho^{2}=.305$

Ring fully plastic:

$Z / k \rho^{2}=.328$ 
It is shown in Ref. [7] that, approximately,

$$
Z / k \rho^{2}=(\rho / R)^{3}\left(\mu K \pi / 2 \rho^{2}\right)
$$

for the elastic ring; here, $K$ is the constant in Eq. (13). The first three equations of Eq. (25) substituted into Eq. (26) with the value of $\rho / R$ appropriate to the size of the elastic core in question give three points on the graph (external force) vs. (axial velocity), and the fourth equation of Eq. (25) gives the value of the asymptote for $K \rightarrow \infty$. This graph is plotted in Fig. 3.

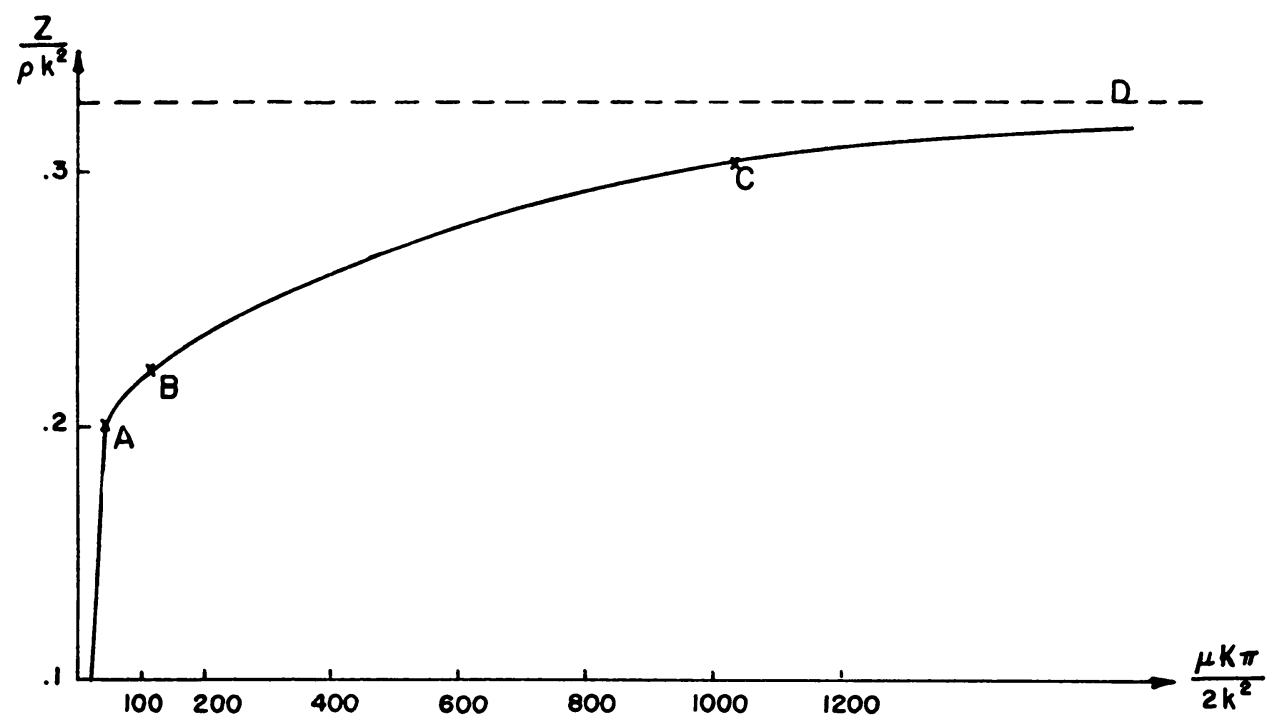

FIG. 3. Resultant force vs. axial velocity. Resultant force: $Z$

Axial velocity: $v_{z}=K \theta$.

$A$ : End of purely elastic deformation

$B$ : Elastic-plastic interface 1 of Fig. 4

$C$ : Elastic-plastic interface 2 of Fig. 4

$D$ : Resultant force for completely plastic ring

Acknowledgements. The solution to the problem of the completely plastic ring was carried out as part of the research program of the Aeronautical Research Laboratories, Melbourne, Australia, and the elastic, plastic solution was found in the course of research sponsored by Watertown Arsenal Laboratories under Contract DA-19-020ORD-2598 with Brown University.

\section{BIBLIOGRAPHY}

1. W. Prager, Discontinuous fields of plastic stress and flow, Proceedings, Second U.S. Congress of Applied Mechanics, Ann Arbor, Mich., 1954, pp. 21-32

2. Jaknke and Emde, Tables of functions, New York, 1938 
3. W. Freiberger, The uniform torsion of a perfectly plastic circular ring, Aeronaut. Research Labs. Rept. SM 213, Melbourne, Australia, 1953

4. A. J. Wang and W. Prager, Plastic twisting of a circular ring sector, J. Mech. Phys. Solids 8, 169-175 (1955)

5. W. Freiberger and W. Prager, Plastic twisting of thick-walled circular ring sectors, J. Appl. Mech. ASME Paper No. 55-A85 (1955)

6. W. Prager and P. G. Hodge, Jr., Theory of perfectly plastic solids, John Wiley and Sons, New York, $1951, \mathrm{p} .73$

7. W. Freiberger, The uniform torsion of an incomplete tore, Australian J. Sci. Research A, 2, 354-375 (1949) 